\title{
Radioimmunotherapy for mantle cell lymphoma: 5-year follow-up of 90 patients from the international RIT registry
}

\author{
Karin Hohloch ${ }^{1,3}(1) \cdot$ Christine Windemuth-Kieselbach $^{2} \cdot$ Pier Luigi Zinzani ${ }^{4} \cdot$ Roberto Cacchione $^{5} \cdot$ Wojciech Jurczak $^{6}$. \\ C. Suh ${ }^{8} \cdot$ Lorenz Trümper $^{3} \cdot$ Christian W. Scholz $^{7}$
}

Received: 15 October 2019 / Accepted: 6 February 2020 / Published online: 3 March 2020

(C) The Author(s) 2020

\begin{abstract}
To assess the efficacy of radioimmunotherapy (RIT) with ${ }^{90}$ yttrium-ibrutinib-tiuxetan (90Y-IT) in mantle cell lymphoma, data from 90 patients registered in the RIT Network with a median follow-up (FU) of 5.5 years after RIT were evaluated. 90Y-IT was given as first-line therapy in $45(50 \%)$ and for relapse in $45(50 \%)$ patients. Most patients received $90 \mathrm{Y}-\mathrm{IT}$ as consolidation after chemoimmunotherapy in first line (98\%) and in relapse (53\%). As a first-line treatment, 30 patients (pts.) (67\%) achieved CR, 10 pts. (22\%) PR\%. and 1 pt. (2\%) PD, and for 4 pts. (9\%), no response data was available. At relapse, CR was achieved in 17 pts. (38\%), PR in 6 pts. (13\%), SD in 2 pts. (4\%), and 6 pts. (13\%) had PD, while the response was not documented for 14 pts. (31\%). After a median FU of 5.5 years, median PFS for all patients was 2.11 (95\% CI, 1.03-2.32) years, and median OS was 4.05 (95\% CI, 2.79-7.21) years. Eleven pts. (12.2\%) developed second malignancy. In conclusion, this is the largest report of MCL pts. treated with $90 \mathrm{Y}$-IT to date. 90Y-IT was most often used as consolidation after first- and second-line chemotherapy and may improve the results achieved using chemoimmunotherapy alone. However, the results are less encouraging compared to treatment with small molecules such as ibrutinib.
\end{abstract}

Keywords Radioimmunotherapy $\cdot$ Mantle cell lymphoma $\cdot$ Outcome

Karin Hohloch

Karin.Hohloch@med.uni-goettingen.de; Karin.Hohloch@ksgr.ch

1 Department of Hematology and Oncology, Kantonsspital Graubünden, Chur, Switzerland

2 Alcedis GmbH, Independent CRO, Giessen, Germany

3 Department of Hematology and Oncology, Georg August University, Goettingen, Germany

4 Institute of Hematology "Seràgnoli”, Università di Bologna, Bologna, Italy

5 Médica e Investigaciones Clínical, "Norberto Quirno", CEMIC, Centro de Educación, Buenos Aires, Argentina

6 Department of Haematology, Jagiellonian University, Cracow, Poland

7 Department of Hematology and Oncology, Vivantes Klinikum Am Urban, Berlin, Germany

8 Asan Medical Center, Department of Internal Medicine, Division of Oncology, University of Ulsan College of Medicine, Seoul, South Korea

\section{Introduction}

Mantle cell lymphoma (MCL) is a radiosensitive disease. Current guidelines recommend involved field radiotherapy (IF-RT) in patients with limited non-bulky stages I and II tumours, preferably as consolidation after a shortened conventional chemotherapy [1]. However, the majority of MCL patients are diagnosed during stage III or IV and are treated with a chemoimmunotherapy, including the anti-CD20 antibody rituximab alongside a chemotherapy, which is chosen according to the performance status of the patient. Younger, more fit patients are treated with induction chemotherapy followed by high-dose chemotherapy (HCT) and autologous stem cell transplantation (ASCT), while less fit individuals receive treatments such as R-CHOP (rituximab, cyclophosphamide, doxorubicin, vincristine, prednisone), R-B (rituximab bendamustine), or R-CAP (rituximab, bortezomib, cyclophosphamide, doxorubicin, prednisone). Recently, addition of rituximab maintenance therapy has led to a survival benefit for patients treated with R-CHOP alone or in combination with HCT and ASCT $[2,3]$. Radioimmunotherapy 
(RIT) with the anti-CD20 antibody ibritumomab connected with tiutexan to the radionucleotide ${ }^{90}$ yttrium combines immuno- and radiotherapy treatment modalities. ${ }^{90}$ yttriumibritumomab-tiuxetan (90Y-IT) (Zevalin $\left.{ }^{\circledR}\right)$ is approved for the treatment of follicular lymphoma, i.e. as consolidation after first-line chemo(immuno)therapy or at relapse. 90Y-IT can be applied in an outpatient setting. Side effects are well managed, most frequently occurring is cytopenia between weeks 6 and 9 after treatment, and infections are not common as well as need for transfusions.

Currently, 90Y-IT is not licensed for the treatment of mantle cell lymphoma (MCL). There are published data from only a few prospective small clinical trials in first $[4,5]$ or later therapy lines [6]. Furthermore, data of long term follow in view of progression-free survival (PFS), overall survival (OS) and secondary malignancies are missing. In 2006, the RIT network (RIT-NT) was founded as a web-based registry using an electronic data capture system for patients B cell non-Hodgkin's lymphoma (NHL) treated with radioimmunotherapy. We show here the results of 90 patients with MCL registered in the RIT-NT treated with 90Y-IT with a medium follow-up of 5.5 years.

\section{Patients and methods}

Between December 2006 and November 2009, the RITNT was active in 14 countries. The RIT-NT evolved from pre-existing national RIT registries in Spain, Austria, Switzerland and Germany. A web-based electronic data capture (EDC) system was utilized for documentation of the patients. The main dataset includes indication, age, haematologic toxicity, lymphoma subtype and clinical course. For the evaluation of specific projects, such as the here presented, datasets from national registries were brought together with the international databases. National approval of the ethics committees (EC) was received by the respective national RIT registry chairs. Written informed consent of patients was mandatory. Data was collected and stored deidentified in the database of the registry. An institutional IRB vote was provided by the EC of the University of Göttingen.

The registry is maintained by a professional clinical research organization (CRO; Alcedis $\mathrm{GmbH}$, Giessen, Germany). Further information regarding the EDC system has been published recently [7]. For the analysis presented here, all initially participating centres with documented mantle cell lymphoma (MCL) patients in the RIT-NT were contacted via mail to take part in the extended follow-up for MCL. For documentation, the web-based electronic data capturing (EDC) system was used.

\section{Results}

Data from 1105 lymphoma patients treated between December 2006 and November 2009 with radioimmunotherapy were documented in the RIT-NT. Out of these 1105 patients, 135 suffered from MCL. Six countries participated with 90 patients in the elongated follow-up analysis (Poland $n=53$, Germany $n=24$, South Korea $n=4$, Italy $\mathrm{n}=4$, Switzerland $n=3$ and Argentina $\mathrm{n}=2$ ), while centres from 8 countries that initially participated in the registry chose not to participate in the report of longer follow-up data for unknown reasons. Follow-up analysis included progressionfree survival, overall survival, relapse therapy and second malignancy.

\section{Patient and disease characteristics}

Median follow-up for the 90 MCL patients treated with 90YIT was 5.5 years (range 0 to 11.5 years). Median age at diagnosis was 63 years (range $31-78$ years), $62 \%$ of patients were older than 60 years, and $21 \%$ were above 70 years. Seventy percent of patients were male, and only $30 \%$ were female (Table 1). Tumour stage was documented only upon initial diagnosis. Using the Ann Arbor classification, 69\% of patients had stage IV, $24 \%$ stage III, $2 \%$ stage II and 3\% stage I disease (Table 1). The MIPI score, bulky disease and extranodal involvement information were not on hand, because neither the MIPI score nor the required variables are incorporated in the documentation of the RIT-NT.

\section{Previous therapies}

In this study, first-line therapy is specified as RIT for primary treatment (monotherapy) or as consolidation after first-line treatment with chemo- or chemoimmunotherapy. Relapse is specified as 90Y-IT given as a monotherapy for relapsed or refractory MCL, as consolidation after chemo(immune)therapy or as section of a conditioning regimen prior to autologous stem cell transplantation. RIT was given as first-line therapy in 45 patients $(50 \%)$ and for relapse in 45 individuals (50\%). In the first-line group, most patients received 90Y-IT as consolidation $(n=44,98 \%)$, while only 1 individual (2\%) was administered 90Y-IT as a monotherapy as first-line treatment. Likewise, in the relapse group, most patients $(n=24,53 \%)$ received RIT as consolidation after immunochemotherapy, 13 pat. received RIT for recurrence, 3 pts. for refractory disease and 2 pts. as part of a conditioning regiment, and for 3 pts., indication for RIT is not documented (Table 2). Of note, 90Y-IT was most often used as first-line therapy $(n=45,50 \%)$ and second-line therapy $(n=23,26 \%)$, while only 20 patients received RIT as third or higher lines of treatment, and for 2 patients, line of therapy was not documented (Table 2). Radiotherapy prior to RIT was infrequent 
Table 1 Patient characteristics

\begin{tabular}{|c|c|c|}
\hline No. of patients & 90 & \\
\hline Male/female & 63 & $(70 \%) / 27(30 \%)$ \\
\hline Median age (range) & 63 & $(31-78)$ \\
\hline$n>60$ years & 56 & $(62.2 \%)$ \\
\hline$n>70$ years & 19 & $(21.1 \%)$ \\
\hline \multirow[t]{2}{*}{ Stage } & No. of patients & $(\%)$ \\
\hline & $n$ & $\%$ \\
\hline Stage IV & 62 & $68.9 \%$ \\
\hline Stage III & 22 & $24.4 \%$ \\
\hline Stage II & 2 & $2.2 \%$ \\
\hline Stage I & 3 & $3.3 \%$ \\
\hline Missing & 1 & $1.1 \%$ \\
\hline \multicolumn{3}{|l|}{ Previous therapies } \\
\hline Chemo(immuno)therapy & $n$ & $\%$ \\
\hline 0 & $4 *$ & $(4.4)$ \\
\hline 1 & $34 *$ & $(37.8)$ \\
\hline 2 & 28 & $(31.1)$ \\
\hline 3 & 11 & $(12.2)$ \\
\hline$>4$ & 13 & (14.4) \\
\hline Radiotherapy & $n$ & $\%$ \\
\hline 0 & 82 & $(91.1)$ \\
\hline 1 & 7 & (7.8) \\
\hline 2 & 1 & $(1.1)$ \\
\hline \multicolumn{3}{|l|}{ Autologous stem cell transplantation } \\
\hline & $n$ & $\%$ \\
\hline & 10 & $(11.11)$ \\
\hline \multicolumn{3}{|l|}{ Indication for RIT } \\
\hline Line & $\mathrm{n}$ & $\%$ \\
\hline First-line therapy & 45 & 50 \\
\hline Primary therapy (RIT mono) & 1 & 1.1 \\
\hline Consolidation (after first-line th.) & 44 & 48.9 \\
\hline Relapse & 45 & 50 \\
\hline Recurrence & 12 & 13.3 \\
\hline Refractory & 3 & 3.3 \\
\hline Conditioning & 2 & 2.2 \\
\hline Consolidation & 24 & 26.7 \\
\hline No indication & 1 & 1.1 \\
\hline Other & 3 & 3.3 \\
\hline
\end{tabular}

with 8 (9\%) patients receiving radiotherapy before RIT. Most patients had $1(n=34,38 \%)$ or $2(n=28,31 \%)$ chemo(immuno)therapies prior to RIT, while 3 chemo(immuno)therapies $(n=11,12 \%), 4(n=7,8 \%), 5$ $(\mathrm{n}=3,3 \%) 6(\mathrm{n}=1,1 \%)$ and more than $7(\mathrm{n}=2,2 \%)$ were less frequent (Table 1). Only 2 pts. had been previously treated with RIT. Of note, in some patients, chemo- and immunotherapy, e.g. COP and rituximab, given as first line, were counted as two therapies. Similarly, omission of one drug from a regimen, e.g. omission of doxorubicin from $\mathrm{CHOP}$ resulting in
CVP, was counted as two therapies in first line in some cases. Therefore, not 44 but only 34 are listed in Table 1 to have received one chemo(immune)therapy before obtaining $90 \mathrm{Y}-$ IT.

At any rate, however, 44 patients received RIT as consolidation after first-line immunochemotherapy, and one patient obtained 90Y-IT without any prior treatment. None of the patients had a Bruton's kinase inhibitor like ibrutinib prior to 90Y-IT.

\section{Response, duration of response and overall survival}

For all patients, response data is only available after RIT. If RIT was preceded by chemoimmunotherapy, which was frequently the case, response was not typically evaluated directly after induction therapy nor was such a response recorded in the RIT registry. Therefore, information regarding conversion, e.g. from PR after induction therapy to CR after RIT, is not available from the RIT registry. For all patients $(n=90)$, the best response was CR in 47 pts. (52\%), PR in 16 pts. (18\%), SD in 2 pts. (2\%) and PD in 7 pts. (8\%). Accurate response data were not reported for $18 \mathrm{pts.}(20 \%)$. In the first-line group with 45 patients, 30 pts. (67\%) achieved complete remission (CR), 10 pts. (22\%) achieved partial remission (PR), 1 pt. (2\%) experienced progressive disease (PD), not documented was response for 4 pts. (9\%). For patients treated in relapse $(n=45)$, the CR rate was $38 \%$, PR was $13 \%$, SD was $4 \%$ and $13 \%$ had PD, while the response was not documented for $31 \%$ $(n=14$ pts.) (Table 3$)$. With a median follow-up of 5.5 years (range $0-11.5$ years), the median PFS for all patients was 2.11 years $(95 \% \mathrm{CI}, 1.03-2.32)$, and median OS was 4.05 years $(95 \%$ CI, 2.79-7.21). For first-line patients, median PFS was 2.79 (95\% CI, 2.14-3.79) years, while it amounted to 0.88 (95\% CI, 0.66-1.5) years for relapsed patients.

Median OS in the first-line group was 4.05 years $(95 \% \mathrm{CI}$, $3.15-7.9)$ and was 3.85 years $(95 \% \mathrm{CI}, 1.49-7.71)$ in the relapse group (Fig. 1).

\section{Second malignancies}

With a median follow-up time of 5.5 years (range 0 11.5 years), in $11(12 \%)$ of the 90 patients, a second malignancy evolved. In nine patients, second malignancy occurred after first-line therapy, and all of these patients had an initial fludarabine-containing regiment (fludarabine, cyclophosphamide [FC], rituximab-FC [R-FC] or R-FC mitoxantrone [RFCM]). In two patients, second malignancy occurred after 5th and 6th line therapy. Time of onset of secondary malignancies after RIT was not documented in the registry. Of the patients with second malignancies, $6(55 \%)$ suffered from myelodysplastic syndrome (MDS), 2 from prostate cancer, 1 from oesophageal cancer, 1 from NSCLC and 1 from rhabdomyosarcoma. 
Table 2 Indication for RIT and line of therapy $(n)$

\begin{tabular}{|c|c|c|c|c|c|c|}
\hline Indication & $\begin{array}{l}\text { Line of therapy } \\
\text { First line }\end{array}$ & Relapse & & & & \\
\hline & First & All & Second & Third & Fourth & $\geq$ Fifth line \\
\hline Conditioning & & 2 & 1 & 1 & & \\
\hline Consolidation & 44 & 24 & 15 & 1 & 2 & 6 \\
\hline Primary therapy & 1 & 0 & & & & \\
\hline Recurrence & & 12 & 5 & 2 & 1 & 4 \\
\hline Refractory & & 3 & 2 & & & 1 \\
\hline Other & & 4 & 1 & 1 & & 1 \\
\hline All & $45(50 \%)$ & $45(50 \%)$ & $24(25.5 \%)$ & $5(5.5 \%)$ & $3(3.3 \%)$ & $12(13.3 \%)$ \\
\hline
\end{tabular}

\section{Discussion}

The RIT registry (RIT-NT) is the largest registry of MCL patients treated with 90Y-IT published to date. Half of the 90 patients reported herein received 90Y-IT as first-line therapy, in most cases as consolidation after chemo- or chemoimmunotherapy. For the remainder, 24 or $26 \%$ of patients were given 90Y-IT as second-line treatment, in most cases (15 of 24 pts.) as consolidation after chemo(immuno)therapy.

Overall response rate and $\mathrm{CR}$ for patients with first-line therapy were $89 \%$ and $67 \%$, respectively. After a median follow-up of 5.5 years, the median PFS and OS for patients treated in first line amounted to 2.79 and 4.05 years, respectively. Toxicity was as expected no unexpected safety signals were detected for employment of 90Y-IT in mantle cell lymphoma. There are few studies employing 90Y-IT as first-line therapy for MCL. In a prospective multicentre trial, 34 patients with MCL were treated as first line with distinct chemo(immuno)therapies (FCM, FC, CHOP or $\mathrm{CVP} \pm \mathrm{R}$ ) and received consolidation with 90Y-IT upon achieving a predefined tumour response after 3 to 6 cycles of treatment. 90Y-IT consolidation improved the CR rate in chemosensitive patients from 41 to $91 \%$, and the median PFS and OS amounted to 3.3 and 6.5 years, respectively [5]. In line with these findings, 57 MCL patients were treated in a prospective singlecentre trial with 90Y-IT if they had achieved at least stable

Table 3 Response according to indication

\begin{tabular}{llll}
\hline Best response & All patients & First line & Relapse \\
\hline & $n(\%)$ & $n(\%)$ & $n(\%)$ \\
CR & $47(52.2)$ & $30(66.7)$ & $17(37.8)$ \\
PR & $16(17.8)$ & $10(22.2)$ & $6(13.3)$ \\
SD & $2(2.2)$ & 0 & $2(4.4)$ \\
PD & $7(7.8)$ & $1(2.2)$ & $6(13.3)$ \\
Missing & $18(20)$ & $4(8.9)$ & $14(31.1)$ \\
\hline
\end{tabular}

disease after four cycles of R-CHOP. Herein, the ORR and $\mathrm{CR}$ rates were $82 \%$ and $52 \%$, respectively, and the median time to treatment failure (TTF) amounted to 34 months [8]. With a longer follow-up median of 9.8 years, median OS for the entire cohort of 56 patients was 7.9 years. During follow-up, one myeloid neoplasia and 6 solid malignancies (2 NSCLC, 1 bladder cancer, 1 ampullary cancer and 2 non-melanoma skin cancers) were observed [4]. These results from 90Y-IT consolidation after shortened chemoimmunotherapy and data from the RIT-NT presented compare well with data from MCL patients treated in clinical trials with six cycles of chemoimmunotherapy with or without rituximab maintenance, i.e. chemoimmunotherapy with R-B (bendamustine), R-CHOP and VR-CAP with or without rituximab maintenance. Here, ORR ranged between 86 and $93 \%$, and the CR rate was between 30 and $53 \%$. Median PFS amounted to 35 months after R-B, ranging from 14 to 22 months after R-CHOP and 24 months after VR-CAP $[9,10]$. Treatment of elderly MCP patients with R-CHOP followed by rituximab maintenance induced a 4year remission duration of approximately $60 \%$ [2]. Therefore, an abbreviated chemoimmunotherapy followed by 90 Y-IT might be just as effective as six cycles of chemoimmunotherapy with or without rituximab maintenance.

In contrast, a phase 2 trial from the Nordic lymphoma group did not show a benefit from applying 90Y-IT to MCL patients who had only achieved PR after induction chemoimmunotherapy with R-maxiCHOP and high-dose cytarabine and were subsequently treated with HCT and ASCT. Here, the data were compared to a previous trial where patients received the same chemoimmunotherapy, including HCT and ASCT, and did not get 90Y-IT. With respect to PFS and OS, adding 90Y-IT did not improve the results [11], indicating that 90Y-IT may not improve CR rates after intensive induction chemoimmunotherapy.

Regarding relapsed MCL in the RIT-NT, the ORR and $\mathrm{CR}$ rates for 90Y-IT as a monotherapy or as consolidation 


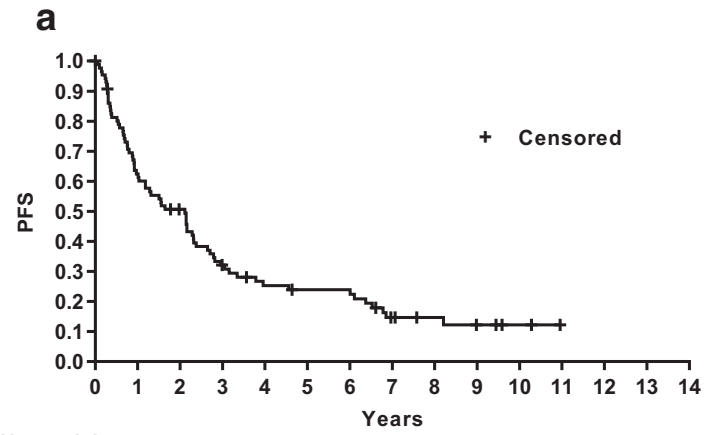

No. at risk:

$\begin{array}{lllllllllll}90 & 53 & 41 & 24 & 18 & 16 & 8 & 6 & 4 & 2 & 0\end{array}$

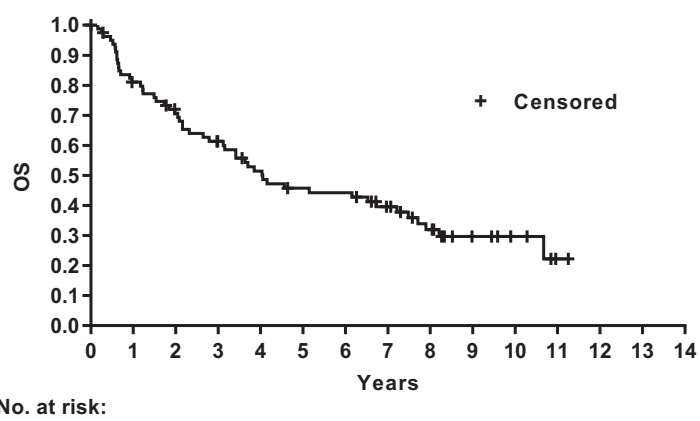

$\begin{array}{lllllllllllll}90 & 63 & 53 & 44 & 36 & 31 & 30 & 23 & 16 & 9 & 5 & 1 & 0\end{array}$ b
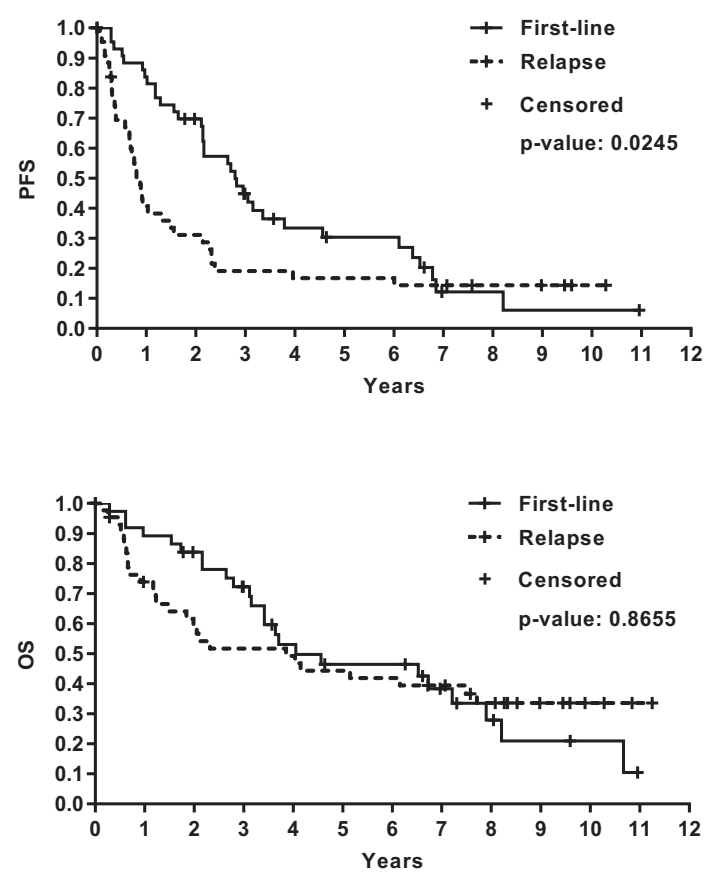

Fig. 1 Progression-free survival (PFS, top) and overall survival (OS, bottom) for all patients (a) or patients on first-line therapy or with relapse (b)

after chemo(immuno)therapy were $51 \%$ and $38 \%$, respectively. Median PFS and OS amounted to 0.88 and 3.85 years, respectively. There are little data from prospective clinical trials employing 90Y-IT in patients with relapsed MCL. In the aforementioned multicentre trial [5], 12 patients received 90Y-IT consolidation after responding to 3-6 cycles of second-line chemo(immune)therapy. Subsequent to 90Y-IT, the CR rate increased from 16 to $75 \%$ in the trial reported by Jurzak et al. [5], resulting in a median PFS and OS of 1.8 and 2.2 years, respectively. In a small trial with relapsed or refractory MCL patients with a median of 3 prior therapies, including high-dose chemotherapy (HCT) and autologous stem cell transplantation (ASCT), 90Y-IT was applied as a monotherapy in 16 patients and as consolidation after chemoimmunotherapy in 32 individuals. The overall response rate and $\mathrm{CR}$ for the entire patient cohort were 61 and $32 \%$, respectively, and the median PFS and OS amounted to 6.2 and 25.5 months, respectively. When 90Y-IT was employed as consolidation, the ORR and CR rates were higher in comparison to 90 Y-IT monotherapy, i.e. ORR 72 compared to $40 \%$ and CR 38 compared to $20 \%$, respectively. The same was true for PFS and OS, i.e. PFS was 8.9 compared to 3.7 months and OS of 31.2 compared to 13.8 months [6]. In a single-centre trial with 90Y-IT monotherapy for patients with relapsed or refractory MCL and a median of 3 prior therapies, the ORR was $31 \%$, CR was $15 \%$, PFS was 6 months, and OS was 21 months. Interestingly, PFS in patients with either CR or PR amounted to 28 months. These data indicate that 90Y-IT may be more effective as consolidation after chemo(immune)therapy and has probably little effect if employed as monotherapy at relapse [12]. Unfortunately, there is no data available from the RIT-NT regarding the response rate prior to $90 \mathrm{Y}$ IT consolidation to strengthen this notion, as response rates were exclusively reported after 90Y-IT.

In line with our data, PFS and OS for the whole group of relapsed or refractory patients amounted to 0.88 (10.8 months) and 3.85 years (46 months). In comparison, the Bruton's kinase inhibitor ibrutinib alone induced a median PFS of 13.9 and 15.8 months after a median of three or two prior therapies and median follow-up of 15.3 months and 39 months, respectively, in two prospective clinical trials [13-15]. Overall survival amounted to $58 \%$ at 18 months and a median of 30.3 months in these two clinical studies, respectively. This demonstrates the efficacy of ibrutinib and underscores its role in relapsed or refractory MCL.

In conclusion, we report results from the largest registry of MCL pts. treated with 90Y-IT to date. Our data and results from prospective clinical trials demonstrate that 90Y-IT containing treatment of mantle cell lymphoma is feasible and achieves results comparable to 
chemoimmunotherapy. Data from clinical trials indicate that 90Y-IT may improve clinical results and may facilitate fewer cycles of chemoimmunotherapy. In our study, as a limitation of the registry data, response rates after induction chemoimmunotherapy, i.e. before 90Y-IT consolidation, were not reported. Therefore, we cannot tell whether 90Y-IT, when given as a consolidation after induction chemoimmunotherapy, improved the response rate achieved by induction treatment. To further elucidate this issue, a prospective clinical trial would be required. Of note, the results with 90Y-IT after chemoimmunotherapy at relapse are far less encouraging compared to treatment with small molecules such as ibrutinib.

Author's contribution Karin Hohloch, Christine Windemuth-Kieselbach: performed the research, data management, and statistics

Christian W. Scholz, Karin Hohloch: designed the study, analysed the data, and wrote the paper

Pire Luigi Zinzani, Roberto Cacchione, Woijciech Jurczak, C.Suh, Lorenz Trümper: draft the paper or revise it critically, approval of the submitted and final version

Funding Information Open Access funding provided by Projekt DEAL.

\section{Compliance with ethical standards}

Conflict of interest The authors declare that they have no conflict of interest.

Open Access This article is licensed under a Creative Commons Attribution 4.0 International License, which permits use, sharing, adaptation, distribution and reproduction in any medium or format, as long as you give appropriate credit to the original author(s) and the source, provide a link to the Creative Commons licence, and indicate if changes were made. The images or other third party material in this article are included in the article's Creative Commons licence, unless indicated otherwise in a credit line to the material. If material is not included in the article's Creative Commons licence and your intended use is not permitted by statutory regulation or exceeds the permitted use, you will need to obtain permission directly from the copyright holder. To view a copy of this licence, visit http://creativecommons.org/licenses/by/4.0/.

\section{References}

1. Dreyling M, Campo E, Hermine O, Jerkeman M, Le Gouill S, Rule S, Shpilberg O, Walewski J, Ladetto M, Committee EG (2017) Newly diagnosed and relapsed mantle cell lymphoma: ESMO clinical practice guidelines for diagnosis, treatment and follow-up. Ann Oncol 28(suppl_4):iv62-iv71. https://doi.org/10.1093/annonc/ $\mathrm{mdx} 223$

2. Kluin-Nelemans HC, Hoster E, Hermine O, Walewski J, Trneny M, Geisler CH, Stilgenbauer S, Thieblemont C, Vehling-Kaiser U, Doorduijn JK, Coiffier B, Forstpointner R, Tilly H, Kanz L, Feugier P, Szymczyk M, Hallek M, Kremers S, Lepeu G, Sanhes L, Zijlstra JM, Bouabdallah R, Lugtenburg PJ, Macro M, Pfreundschuh M, Prochazka V, Di Raimondo F, Ribrag V, Uppenkamp M, Andre M, Klapper W, Hiddemann W, Unterhalt M, Dreyling MH (2012) Treatment of older patients with mantle- cell lymphoma. N Engl J Med 367(6):520-531. https://doi.org/10. 1056/NEJMoa1200920

3. Le Gouill S, Thieblemont C, Oberic L, Moreau A, Bouabdallah K, Dartigeas C, Damaj G, Gastinne T, Ribrag V, Feugier P, Casasnovas O, Zerazhi H, Haioun C, Maisonneuve H, Houot R, Jardin F, Van Den Neste E, Tournilhac O, Le Du K, Morschhauser F, Cartron G, Fornecker LM, Canioni D, Callanan M, Bene MC, Salles G, Tilly H, Lamy T, Gressin R, Hermine O, Group L (2017) Rituximab after autologous stem-cell transplantation in mantle-cell lymphoma. $\mathrm{N}$ Eng1 J Med 377(13):1250-1260. https://doi.org/10.1056/ NEJMoa1701769

4. Smith MR, Hong F, Li H, Gordon LI, Gascoyne RD, Paietta EM, Advani RH, Forero-Torres A, Horning SJ, Kahl BS (2017) Mantle cell lymphoma initial therapy with abbreviated R-CHOP followed by (90)Y-ibritumomab tiuxetan: 10-year follow-up of the phase 2 ECOG-ACRIN study E1499. Leukemia 31(2):517-519. https://doi. org/10.1038/leu.2016.305

5. Jurczak W, Gruszka AM, Sowa Staszczak A, Dlugosz-Danecka M, Szostek M, Zimowska-Curylo D, Giza A, Krawczyk K, Jakobczyk M, Hubalewska-Dydejczyk A, Szymczyk M, Wrobel T, Knopinska-Posluszny W, Kisiel E, Skotnicki A, Zinzani PL (2019) Consolidation with (90)Y ibritumomab tiuxetan radioimmunotherapy in mantle cell lymphoma patients ineligible for high dose therapy: results of the phase II multicentre polish lymphoma research group trial, after 8-year long follow-up. Leuk Lymphoma:1-8. https://doi.org/10.1080/10428194.2019.1602261

6. Ferrero S, Pastore A, Scholz CW, Forstpointner R, Pezzutto A, Bergmann L, Trumper L, Finke J, Keller U, Ghione P, Passera R, Hiddemann W, Weigert O, Unterhalt M, Dreyling M (2016) Radioimmunotherapy in relapsed/refractory mantle cell lymphoma patients: final results of a European MCL network phase II trial. Leukemia 30(4):984-987. https://doi.org/10.1038/leu.2015.215

7. Hohloch K, Windemuth-Kieselbach C, Kolz J, Zinzani PL, Cacchione R, Jurczak W, Bischof Delaloye A, Trumper L, Scholz CW (2019) Radioimmunotherapy (RIT) for follicular lymphoma achieves long term lymphoma control in first line and at relapse: 8-year follow-up data of 281 patients from the international RITregistry. Br J Haematol 184(6):949-956. https://doi.org/10.1111/ bjh. 15712

8. Smith MR, Li H, Gordon L, Gascoyne RD, Paietta E, Forero-Torres A, Kahl BS, Advani R, Hong F, Horning SJ (2012) Phase II study of rituximab plus cyclophosphamide, doxorubicin, vincristine, and prednisone immunochemotherapy followed by yttrium-90ibritumomab tiuxetan in untreated mantle-cell lymphoma: eastern cooperative oncology group study E1499. J Clin Oncol 30(25): 3119-3126. https://doi.org/10.1200/JCO.2012.42.2444

9. Rummel MJ, Niederle N, Maschmeyer G, Banat GA, von GU LC, Kofahl-Krause D, Heil G, Welslau M, Balser C, Kaiser U, Weidmann E, Durk H, Ballo H, Stauch M, Roller F, Barth J, Hoelzer D, Hinke A, Brugger W (2013) Bendamustine plus rituximab versus $\mathrm{CHOP}$ plus rituximab as first-line treatment for patients with indolent and mantle-cell lymphomas: an open-label, multicentre, randomised, phase 3 non-inferiority trial. Lancet 381(9873):1203-1210. https://doi.org/10.1016/S0140-6736(12) $61763-2$

10. Robak T, Huang H, Jin J, Zhu J, Liu T, Samoilova O, Pylypenko H, Verhoef G, Siritanaratkul N, Osmanov E, Alexeeva J, Pereira J, Drach J, Mayer J, Hong X, Okamoto R, Pei L, Rooney B, van de Velde H, Cavalli F, Investigators LYM (2015) Bortezomib-based therapy for newly diagnosed mantle-cell lymphoma. N Engl J Med 372(10):944-953. https://doi.org/10.1056/NEJMoa1412096

11. Kolstad A, Laurell A, Jerkeman M, Gronbaek K, Elonen E, Raty R, Pedersen LB, Loft A, Bogsrud TV, Kimby E, Hansen PB, Fagerli UM, Nilsson-Ehle H, Lauritzsen GF, Lehmann AK, Sundstrom C, Karjalainen-Lindsberg ML, Ralfkiaer E, Ehinger M, Delabie J, Bentzen H, Schildt J, Kostova-Aherdan K, Frederiksen H, Brown 
Pde N, Geisler CH, Nordic Lymphoma G (2014) Nordic MCL3 study: 90Y-ibritumomab-tiuxetan added to BEAM/C in non-CR patients before transplant in mantle cell lymphoma. Blood 123(19):2953-2959. https://doi.org/10.1182/blood-2013-12541953

12. Wang M, Oki Y, Pro B, Romaguera JE, Rodriguez MA, Samaniego F, McLaughlin P, Hagemeister F, Neelapu S, Copeland A, Samuels BI, Loyer EM, Ji Y, Younes A (2009) Phase II study of yttrium-90ibritumomab tiuxetan in patients with relapsed or refractory mantle cell lymphoma. J Clin Oncol 27(31):5213-5218. https://doi.org/10. 1200/JCO.2009.21.8545

13. Wang ML, Rule S, Martin P, Goy A, Auer R, Kahl BS, Jurczak W, Advani RH, Romaguera JE, Williams ME, Barrientos JC, Chmielowska E, Radford J, Stilgenbauer S, Dreyling M, Jedrzejczak WW, Johnson P, Spurgeon SE, Li L, Zhang L, Newberry K, Ou Z, Cheng N, Fang B, McGreivy J, Clow F, Buggy JJ, Chang BY, Beaupre DM, Kunkel LA, Blum KA (2013) Targeting BTK with ibrutinib in relapsed or refractory mantle-cell lymphoma. N Engl J Med 369(6):507-516. https:// doi.org/10.1056/NEJMoa1306220
14. Dreyling M, Jurczak W, Jerkeman M, Silva RS, Rusconi C, Trneny M, Offner F, Caballero D, Joao C, Witzens-Harig M, Hess G, Bence-Bruckler I, Cho SG, Bothos J, Goldberg JD, Enny C, Traina S, Balasubramanian S, Bandyopadhyay N, Sun S, Vermeulen J, Rizo A, Rule S (2016) Ibrutinib versus temsirolimus in patients with relapsed or refractory mantle-cell lymphoma: an international, randomised, open-label, phase 3 study. Lancet 387(10020):770-778. https://doi.org/10.1016/S0140-6736(15) 00667-4

15. Rule S, Jurczak W, Jerkeman M, Rusconi C, Trneny M, Offner F, Caballero D, Joao C, Witzens-Harig M, Hess G, Bence-Bruckler I, Cho SG, Thieblemont C, Zhou W, Henninger T, Goldberg J, Vermeulen J, Dreyling M (2018) Ibrutinib versus temsirolimus: 3year follow-up of patients with previously treated mantle cell lymphoma from the phase 3, international, randomized, open-label RAY study. Leukemia 32(8):1799-1803. https://doi.org/10.1038/ s41375-018-0023-2

Publisher's note Springer Nature remains neutral with regard to jurisdictional claims in published maps and institutional affiliations. 